\title{
Pannexin 1 Differentially Affects Neural Precursor Cell Maintenance in the Ventricular Zone and Peri-Infarct Cortex
}

\author{
닐 Leigh E. Wicki-Stordeur, ${ }^{1}$ J Juan C. Sanchez-Arias, ${ }^{1}$ Jagroop Dhaliwal, ${ }^{2}$ Esther 0. Carmona-Wagner, ${ }^{1}$ \\ - Valery I. Shestopalov, ${ }^{3,4}$ Diane C. Lagace, ${ }^{2}$ and Leigh Anne Swayne ${ }^{1,5}$ \\ ${ }^{1}$ Division of Medical Sciences, University of Victoria, Victoria, British Columbia V8P 5C2, Canada, ${ }^{2}$ Department of Cellular and Molecular Medicine, \\ University of Ottawa, Ottawa, Ontario K1H 8M5, Canada, ${ }^{3}$ Bascom Palmer Eye Institute, Department of Ophthalmology, University of Miami Miller School \\ of Medicine, Miami, Florida 33136, ${ }^{4}$ Vavilov Institute of General Genetics RAS, Moscow, Russian Federation 119333, and ${ }^{5}$ Island Medical Program and \\ Department of Cellular and Physiological Sciences, University of British Columbia, Vancouver, British Columbia V6T 1Z3, Canada
}

We demonstrated previously that Pannexin 1 (Panx1), an ion and metabolite channel, promotes the growth and proliferation of ventricular zone (VZ) neural precursor cells (NPCs) in vitro. To investigate its role in vivo, we used floxed Panx1 mice in combination with viruses to delete Panx1 in VZ NPCs and to track numbers of Panx1-null and Panx1-expressing VZ NPCs over time. Two days after virus injection, Panx1-null cells were less abundant than Panx1-expressing cells, suggesting that Panx1 is required for the maintenance of VZ NPCs. We also investigated the effect of Panx1 deletion in VZ NPCs after focal cortical stroke via photothrombosis. Panx1 is essential for maintaining elevated VZ NPC numbers after stroke. In contrast, Panx1-null NPCs were more abundant than Panx1-expressing NPCs in the peri-infarct cortex. Together, these findings suggest that Panxl plays an important role in NPC maintenance in the VZ niche in the naive and stroke brain and could be a key target for improving NPC survival in the peri-infarct cortex.

Key words: neural precursor; pannexin; stroke

Significance Statement

Here, we demonstrate that Pannexin 1 (Panx1) maintains a consistent population size of neural precursor cells in the ventricular zone, both in the healthy brain and in the context of stroke. In contrast, Panxl appears to be detrimental to the survival of neural precursor cells that surround damaged cortical tissue in the stroke brain. This suggests that targeting Panxl in the peri-infarct cortex, in combination with other therapies, could improve cell survival around the injury site.

\section{Introduction}

Pannexins (Panx1, Panx2, and Panx3) form channels that are permeable to ions and small metabolites such as ATP (Bao et al., 2004). They were discovered based on their homology to the gap junction forming proteins in invertebrates, the innexins (Pan-

\footnotetext{
Received Feb. 1, 2015; revised Nov. 20, 2015; accepted Dec. 15, 2015.

Author contributions: L.E.W.-S., J.D., V.I.S., D.C.L., and L.A.S. designed research; L.E.W.-S., J.C.S.-A., J.D., E.O.C.W., D.C.L., and L.A.S. performed research; L.E.W.-S., J.C.S.-A., E.O.C.-W., D.C.L., and L.A.S. analyzed data; L.E.W.-S., V.I.S., D.C.L., and L.A.S. wrote the paper.

This work was supported by grants from the Heart and Stroke Foundation Canadian Partnership for Stroke Recovery (CPSR Expansion Grant to L.A.S. and D.C.L.), the Natural Sciences and Engineering Research Council of Canada (NSERC Discovery Grant to L.A.S.), the Canadian Institutes of Health Research (Grant MOP142215 to L.A.S.), and the University of Victoria Division of Medical Sciences (Seed Grant to L.A.S.). L.A.S. is supported by a Michael Smith Foundation for Health Research and a British Columbia Schizophrenia Society Foundation Scholar Award. L.E.W.-S. is supported by a Vanier Canada Graduate Scholarship (NSERC) and an Edythe Hembroff-Schleicher Scholarship. V.I.S. was supported by the National Eye Institute-National Institutes of Health (Grants EY021517, P30 EY014801, and RPB unrestricted Grant to the University of Miami Department of Ophthalmology). We thank Mirela Hasu, Angela Nguyen, Keren Leviel Kumar (University of Ottawa), and Anthony Carter (CPSR) for technical support and the Canadian Foundation for Innovation and the British Columbia Knowledge Development Fund for granting funds to L.A.S. for our Leica SP8 confocal microscope.

The authors declare no competing financial interests.
}

chin et al., 2000), but form primarily single-membrane channels (for review, see Sosinsky et al., 2011). Panxl is enriched in the nervous system and was originally detected in mature neurons (Ray et al., 2005; Vogt et al., 2005). Panx1 channels are activated by mechanical stimulation, membrane depolarization, increased extracellular $\mathrm{K}^{+}$, oxygen-glucose deprivation, and caspase cleavage (Bruzzone et al., 2003; Bao et al., 2004; Locovei et al., 2006; Thompson et al., 2006; Ma et al., 2009; Silverman et al., 2009; Chekeni et al., 2010; Santiago et al., 2011).

Recently, we discovered Panx1 expression in postnatal neural precursor cells (NPCs) of the ventricular zone (VZ) (WickiStordeur et al., 2012; Wicki-Stordeur and Swayne, 2013). VZ NPCs continually undergo proliferation, differentiation, and migration through the rostral migratory stream (RMS) (for review, see Ming and Song, 2011). Along this journey, a large proportion

Correspondence should be addressed to Leigh Anne Swayne, Division of Medical Sciences, Medical Sciences Building, Rm 224, University of Victoria, 3800 Finnerty Rd, Victoria, BC V8P 5C2, Canada. E-mail: Iswayne@uvic.ca. DOl:10.1523/JNEUROSCI.0436-15.2016

Copyright $\odot 2016$ the authors $\quad 0270-6474 / 16 / 361203-08 \$ 15.00 / 0$ 
are lost (Morshead and van der Kooy, 1992), whereas the surviving NPCs become resident postmitotic neurons in the olfactory bulb. These cells are important in olfactory-associated learning and memory (Mak and Weiss, 2010; Sakamoto et al., 2014). We found that Panx1 promotes VZ NPC proliferation in vitro (Wicki-Stordeur et al., 2012). In the present study, we investigated the impact of Panx1 deletion on the number of VZ NPCs in vivo over time. We used floxed Panx1 mice injected intracerebroventricularly with control and Crerecombinase retroviruses coexpressing different fluorescent markers (Tashiro et al., 2006a; Tashiro et al., 2006b) to track both Panx1-null and Panx1-expressing NPC numbers over time, essentially a measure of NPC "maintenance." In addition, because focal cortical ischemia is well known to activate VZ NPCs to increase their proliferation rate (for review, see Ohab and Carmichael, 2008) and because Panxl has been strongly associated with stroke (Thompson et al., 2006; Bargiotas et al., 2011; Bargiotas et al., 2012; Dvoriantchikova et al., 2012; Xiong et al., 2014) and inflammation (for review, see Makarenkova and Shestopalov, 2014), we also investigated the impact of Panx1 deletion on VZ NPC numbers in the context of stroke both in the $\mathrm{VZ}$ and in the peri-infarct cortex.

Overall, our results suggested that the presence of Panx1 was differentially important for the maintenance of NPCs depending on their location. The deletion of Panx1 impaired NPC maintenance in the VZ niche. In the context of stroke, which stimulates NPC proliferation, the effect of Panx1 deletion was similar but significantly delayed. In contrast, maintenance of NPCs in the peri-infarct cortex (that had migrated from the VZ) was improved by Panx1 deletion. Together, these findings represent important first steps in examining the NPC-specific role of Panx1 in the healthy brain and in the context of stroke.

\section{Materials and Methods}

Animals. All procedures were performed in agreement with the guidelines of the Canadian Council for Animal Care and the University of Victoria and University of Ottawa Animal Care Committees. Focal cortical ischemia was induced by photothrombosis of the cortical microvasculature (as described in Watson et al., 1985). Briefly, adult (2-4 months) "floxed" Panx1-LoxP mice (on a 129 background confirmed by genotyping; Dvoriantchikova et al., 2012) were anesthetized using isofluorane and maintained at $37^{\circ} \mathrm{C}$ with a heating pad. A $1 \%$ Rose Bengal (Sigma-Aldrich) solution (in brain buffer: $0.04 \mathrm{M} \mathrm{NaH}_{2} \mathrm{PO}_{4}, 0.16 \mathrm{M}$ $\mathrm{Na}_{2} \mathrm{HPO}_{4}$ ) was injected intraperitoneally $2-5$ min before laser illumination. The skull was exposed by a midline incision and a site $2.25 \mathrm{~mm}$ left of the midline and $0.7 \mathrm{~mm}$ anterior to bregma was illuminated for $10 \mathrm{~min}$ by a laser calibrated to $532 \mathrm{~nm}$. Retrovirus was used to target primarily late-stage NPCs (Tashiro et al., 2006a; Tashiro et al., 2006b) in the VZ. CAG-red fluorescent protein (RFP) and CAG-green fluorescent protein (GFP)-Cre viruses were mixed in a 1:1 ratio and injected bilaterally at the time of stroke at coordinates $1.2 \mathrm{~mm}$ right and left of the midline, $1.0 \mathrm{~mm}$ posterior to bregma, and $1.9 \mathrm{~mm}$ in depth. Mice were killed at 2, 5, or $10 \mathrm{~d}$ postinjection/photothrombosis (dpi/PT) $[n=7$ ( 4 male, 3 female) for 2 dpi/PT and $n=6$ for $5 \mathrm{dpi} / \mathrm{PT}$ ( 2 male, 4 female) and $10 \mathrm{dpi} / \mathrm{PT}$ ( 4 male, 2 female)]. Naive floxed Panx 1 mice were given bilateral virus injection without stroke, and killed at 2 or $10 \mathrm{dpi}[n=5$ ( 3 male, 2 female) for $2 \mathrm{dpi}$ and $n=6$ ( 3 male, 3 female) for 10 dpi]. Naive wild-type 129 control mice were given bilateral virus injection without stroke and killed at $2 \mathrm{dpi}$ [ $n=7$ (4 male, 3 female) .

Microscopy. Mouse brain cryopreservation and serial cryosectioning were performed as described previously (Swayne et al., 2010; WickiStordeur et al., 2012). Antibodies were diluted in $10 \mathrm{~mm}$ PBS supplemented with $0.3 \%$ Triton X-100 and $3 \%$ bovine serum albumin. Confocal immunofluorescence imaging was performed as described previously (Wicki-Stordeur et al., 2012, 2013; Wicki-Stordeur and Swayne, 2013) using a Leica SP8 confocal microscope. In general, representative images were produced with Adobe Photoshop CS5 Extended software and uniformly adjusted for brightness/contrast.

For virus quantifications, $600 \times 400 \mu \mathrm{m}$ boxes were drawn around the VZ dorsolateral corner, the ventral boundary of the stroke, and the medial edge of the stroke boundary and aligned with the pial surface as shown in the figures. In addition, representative coronal slices from each animal were taken for virus quantifications in the RMS (see Fig. 1). Our area of quantification was equivalent between animals and was restricted to the circle/oval of condensed nuclei rostral to the opening of the lateral ventricles. Hoechst 33342 was used as a nuclear counterstain in all images. RFP fluorescence was present in both cytoplasmic and nuclear compartments of the NPCs, whereas GFP signal was localized to the nucleus. Therefore, our VZ and RMS counting criteria were that a positive cell must have GFP and/or RFP signal overlapping with a Hoechst-positive nucleus. Panx1-expressing NPCs possessed RFP fluorescence only and Panx1-null NPCs had nuclear GFP fluorescence with or without RFP fluorescence. Quantification of transduced NPCs in the VZ of wild-type 129 control mice revealed relatively equal expected populations of RFPpositive only and GFP-positive NPCs per VZ ( $45 \%$ vs $55 \%$, each $\pm 2 \%$; $n=7,2$ dpi). We saw no significant differences in NPC labeling between hemispheres in stroke animals and therefore presented pooled contralateral and ipsilateral data for each subsequent analysis. Data are presented as mean number of NPCs per VZ or RMS quantification region (outlined above). The data from each individual animal was considered as an independent biological replicate.

For lineage analysis, images of equal area were taken from the dorsolateral corner of the VZ and overlap between Cre-GFP or RFP and DCX signal was analyzed. Our counting criteria were such that a transduced cell was considered DCX-positive if $2 / 3$ of its surface was surrounded by DCX signal in at least 1 plane of a confocal $z$-stack.

For proliferation analysis, images of equal area were taken from the dorsolateral corner of the VZ and overlap between Cre-GFP or RFP and Ki67 signal was analyzed. Our counting criteria were such that a transduced cell was considered Ki67-positive if the corresponding nucleus overlapped with Ki67 signal in at least one plane of a confocal $z$-stack.

For apoptosis analysis, images of equal area were taken from the periinfact cortex (as described above) and overlap between Cre-GFP or RFP and activated caspase $3\left({ }^{\star}\right.$ Casp 3$)$ was analyzed. Our counting criteria were such that a transduced cell was considered ${ }^{\star}$ Casp3-positive if the corresponding nucleus overlapped with ${ }^{\star}$ Casp3 signal in at least one plane of a confocal $z$-stack.

Antibodies. Primary antibodies used were as follows: anti-doublecortin (DCX; 1:1000; Millipore), anti-Ki67 (1:200; BD Biosciences), and anticleaved caspase 3 (1:3000; Cell Signaling Technology). Secondary antibodies used were Alexa Fluor 647-conjugated AffiniPure donkey anti-rabbit IgG, DyLight 405-conjugated AffiniPure donkey anti-guinea pig IgG, and DyLight 649-conjugated AffiniPure donkey anti-mouse IgG (all 1:300; all from Jackson ImmuoResearch).

Statistical analysis. Statistical analyses were performed using Prism for Mac OS X version 5.0d software (GraphPad). Statistical tests are reported in each figure legend. For ANOVA's the "expression" factor refers to the Panxl expression status of the transduced cells (Panx1-expressing vs Panx1-null). All variances are reported as SEM. Significance was denoted as $\left.p<0.05\left(^{*}\right), p<0.01{ }^{* *}\right)$. Exact $p$-values are provided in the figure legends.

\section{Results}

\section{Panx1 is required for maintenance of VZ NPCs}

We demonstrated previously the presence of Panx1 in VZ NPCs and their progeny (Wicki-Stordeur et al., 2012; Wicki-Stordeur and Swayne, 2013) and showed that Panx1 promoted VZ NPC proliferation in vitro (Wicki-Stordeur et al., 2012). We therefore predicted that Panx1 is important for the regulation of VZ NPCs in vivo. To investigate this hypothesis, we used a retrovirus strategy to genetically ablate Panx1 in VZ NPCs (Tashiro et al., 2006a; Tashiro et al., 2006b). In this approach, a combination of CreGFP/RFP-control retroviruses was injected by intracerebroventricular injection into floxed Panx1 mice in a 1:1 ratio (Fig. 1A). 


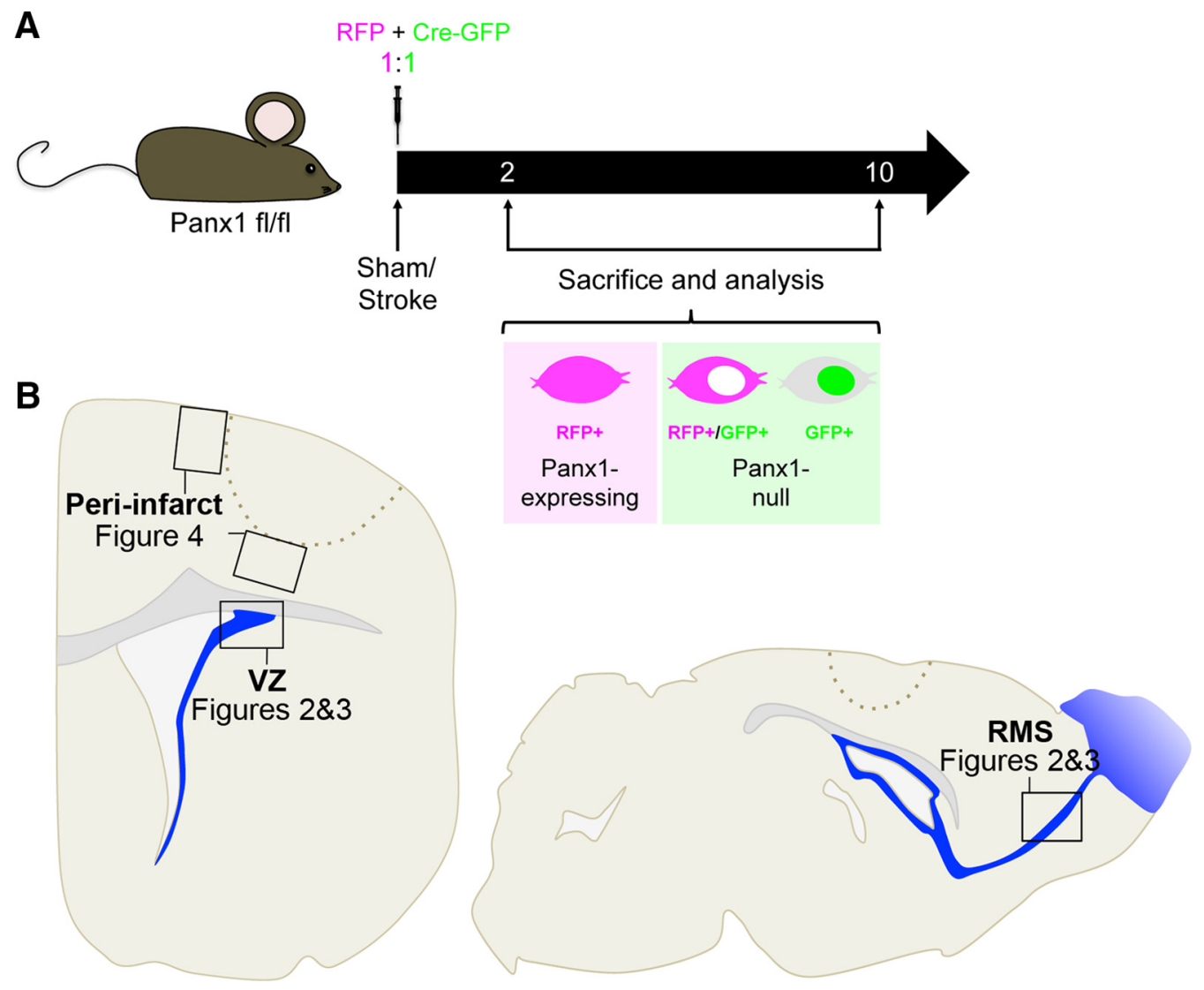

Figure 1. Experimental outline for retrovirus-mediated Panx1 deletion in VZ NPCs in naive/sham and stroke mice. A, Panx1-LoxP mice were given intracerebroventricular injections of retroviral particles to introduce Cre-GFP or RFP-control vectors (1:1 ratio). Naive/sham and photothrombotic stroke conditions were examined 2 and $10 \mathrm{~d}$ after surgery. Panx1-expressing (RFP ${ }^{+}$only) and Panx1-null (Cre-GFP ${ }^{+}$and $\mathrm{Cre}_{\text {GFP }}{ }^{+} /$RFP $^{+}$) NPCs were counted. RFP signal was present throughout the cell, whereas Cre-GFP signal was localized to the nucleus. $\boldsymbol{B}$, Diagram representing areas of quantification in the VZ, RMS, and peri-infarct cortex. Labels refer to the figures in which the corresponding data can be found.

As outlined in the diagram in Figure $1 B$, in the course of this study, we investigated both naive and stroke conditions and quantified the fluorescently labeled NPCs in the dorsolateral corner of the VZ, the RMS, and the peri-infarct cortex. We used the quantification of the number of labeled NPCs over time as a metric for maintenance (i.e., the preservation of a consistent population size). Because naive animals underwent a similar surgical procedure for virus injection (without dye injection/laser illumination), they were considered as sham controls for stroke surgery (henceforth referred to as "naive/sham").

We introduced the retrovirus mixture into the floxed Panx1 strain (naive/sham animals) and counted the number of Panx1null and Panx1-expressing NPCs in the VZ (Fig. 2A). Prior work in wild-type mice (see Materials and Methods) established that equally sized populations of GFP-positive and RFP-positive only NPCs per VZ were expected if Panxl deletion had no effect. However, initially (2 dpi), there were $~ 70 \%$ less Panx1-null NPCs (GFP-positive) than Panx1-expressing NPCs (RFPpositive only). Over time, the number of Panx1-expressing NPCs decreased (and there was no statistically significant change in the number of Panx1-null NPCs) such that, by $10 \mathrm{dpi}$, there was no significant difference between Panx1-null and Panx1-expressing NPCs. We confirmed that virtually all of the transduced NPCs (both Panx1-null and Panx1-expressing) 2 dpi were positive for DCX (Fig. 2B), a marker for late stage NPCs (neuroblasts) and immature neurons (for review, see Ming and Song, 2011).

We reasoned that a defect in proliferation associated with Panx1 deletion could have caused the lower abundance of Panx1- null NPCs. To examine the proliferation status of infected NPCs, we immunostained for Ki67 (Fig. 2C), a marker of actively cycling cells (for review, see Scholzen and Gerdes, 2000). The percentage of Ki67-positive NPCs was independent of Panx1 expression status. Another possible explanation for the loss of Panx1-null NPCs in the VZ could be accelerated migration out of the VZ into the RMS. However, the number of Panx1-null NPCs was low in the RMS (Fig. 2D), ruling out this possibility. We also immunostained for activated caspase 3, a marker for cells undergoing apoptosis (for review, see Thornberry and Lazebnik, 1998). We did not detect any activated caspase 3-positive cells in the VZ. Together, these results suggest that Panx1 is essential for maintenance of VZ NPCs, but does not affect proliferation, migration, or caspase 3-dependent apoptotic mechanisms in vivo.

\section{Stroke delays the effect of Panx1 deletion on VZ NPC maintenance}

VZ NPCs are activated by cortical stroke to hyperproliferate despite their distance from the injury site (for review, see Ohab and Carmichael, 2008) and Panxl is activated by stimuli associated with stroke (Thompson et al., 2006; Silverman et al., 2009; Weilinger et al., 2012). We therefore investigated whether cortical stroke alters the effects of Panx1 deletion on VZ NPC maintenance using the photothrombotic (PT) model (Fig. 3). Note that virus injection was performed during the same surgical procedure.

Stroke increased the total numbers of infected NPCs (GFP- and RFP-positive populations combined) at $2 \mathrm{dpi}$ (naive/sham: $11.9 \pm$ 

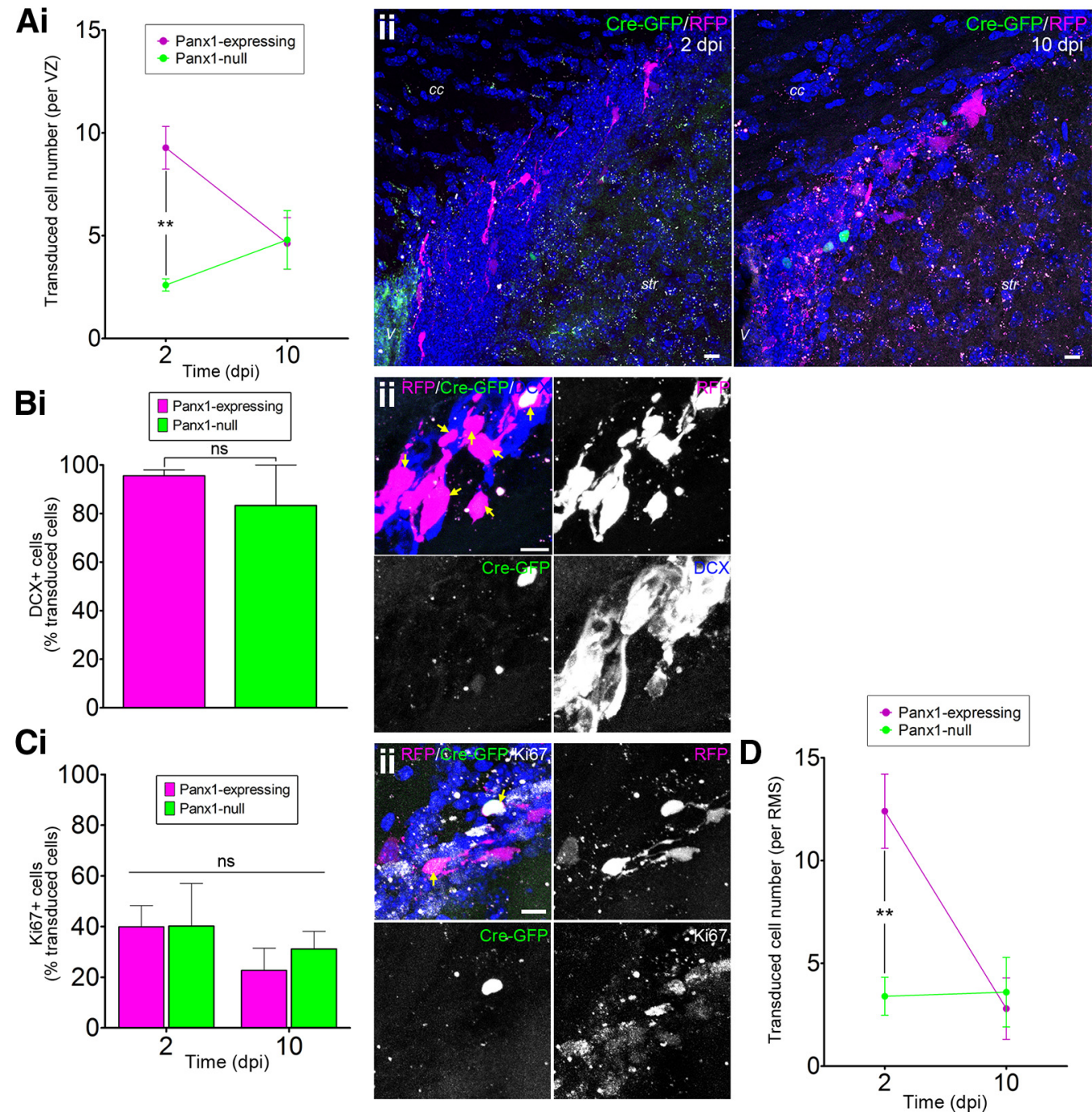

Figure 2. Panx1 deletion is associated with a loss of VZ NPCs. Ai, Panx1-null (GFP ${ }^{+}$) and Panx1-expressing (RFP ${ }^{+}$only) NPC numbers per VZ at 2 and 10 dpi in naive/sham animals. The number of Panx1-null NPCs was lower than Panx1-expressing NPCs at $2 \mathrm{dpi}\left(2 \mathrm{dpi}, n=5 ; 10 \mathrm{dpi}, n=6\right.$; expression: $F_{(1,18)}=7.898, p=0.0116$; time: $F_{(1,18)}=1.123, p=0.3033$; interaction: $F_{(1,18)}=$ $8.764, p<=0.0084$; by 2 -factor ANOVA; Bonferroni post hoc ${ }^{* *} p<0.01$ for expression at 2 dpi). Aii, Maximum-intensity projections of representative confocal Z-stacks of the VZ at 2 (left) and $10 \mathrm{dpi}$ (right). Scale bars, $10 \mu \mathrm{m}$. V, Ventricle; cc, corpus callosum, str, striatum. Hoechst 33342 was used as a nuclear counterstain. Bi, The vast majority of Panx1-expressing and Panx1-null VZNPCs were immunoreactive for $D C X(2$ dpi, $n=6, p=0.3930$ by unpaired $t$ test). Bii, Maximum-intensity projection of a representative confocal Z-stack demonstrating $D C X$ immunoreactivity of transduced VZ NPCs. Scale bar, $10 \mu \mathrm{m}$. Arrows indicate DCX ${ }^{+}$transduced cells. Ci, The percentage of transduced VZ NPCs immunoreactive for Ki67 was not affected by time after injection or Panx 1 expression status ( $2 \mathrm{dpi}, n=4 ; 10 \mathrm{dpi}, n=6$; expression: $F_{(1,16)}=0.1885, p=0.6699$; time: $F_{(1,16)}=1.633, p=0.2195$; interaction: $F_{(1,16)}=0.1582, p=0.6961$ by 2-factor ANOVA). Cii, Maximum-intensity projection of a representative confocal Z-stack from the VZ showing Ki67-immunoreactivity of transduced VZ NPCs. Scale bar, $10 \mu \mathrm{m}$. Arrows indicate Ki67 ${ }^{+}$transduced NPCs. Hoechst 33342 was used as a nuclear counterstain. $D$, Quantification of transduced NPC numbers in the RMS at 2 and $10 \mathrm{dpi}\left(2 \mathrm{dpi}, n=5 ; 10 \mathrm{dpi}, n=6\right.$; expression: $F_{(1,16)}=7.293, p=0.0158$; time: $F_{(1,16)}=9.584, p=0.0069$; interaction: $F_{(1,16)}=10.42, p=0.0053$ by 2 -factor ANOVA; Bonferroni post hoc ${ }^{* *} p<0.01$ for expression at 2 dpi).

1.3 , stroke: $41.5 \pm 10.1$ transduced cells/VZ, $p=0.0264$ by unpaired $t$ test), which was expected because the population of DCX-positive NPCs has been reported to increase in response to cortical stroke (for review, see Ohab and Carmichael, 2008). At 2 dpi/PT, Panx1-null and Panx1-expressing NPCs were equally abundant (no significant difference by two-factor ANOVA) in the VZ (Fig. $3 A$ ) and virtually all transduced cells were DCX-positive (Fig. 3B). However, by 10 dpi/PT, the number of Panx1-null NPCs was significantly reduced to naive/sham levels, whereas the number of Panx1-expressing NPCs remained elevated. We added an intermediate time point (5 $\mathrm{dpi} / \mathrm{PT}$ ) to gain further insight into the dynamics of this reduction in Panx1-null NPCs. The Panx1-null NPC numbers were already largely (albeit not significantly) reduced by $5 \mathrm{dpi} / \mathrm{PT}$.

The percentage of Ki67-positive NPCs was independent of Panx1 expression status (Fig. 3C), suggesting that the loss of
Panx1-null NPCs was not due to a reduction in proliferation. Furthermore, the abundance of labeled NPCs in the RMS was independent of Panx1 expression status (Fig. 3D), suggesting that there was no effect of Panxl deletion on migration. We also immunostained for activated caspase 3, a marker for cells undergoing apoptosis, and again did not detect any activated caspase 3-positive cells in the VZ. Together, these results suggest that Panx1 is also essential for maintaining elevated VZ NPC numbers after stroke and does not affect proliferation, migration, or caspase 3-dependent apoptotic mechanisms.

\section{Panx1-null NPCs are more abundant in the} peri-infarct cortex

We also hypothesized that deletion of Panx1 could influence the survival of VZ NPCs that migrate into the peri-infarct cortex (for 

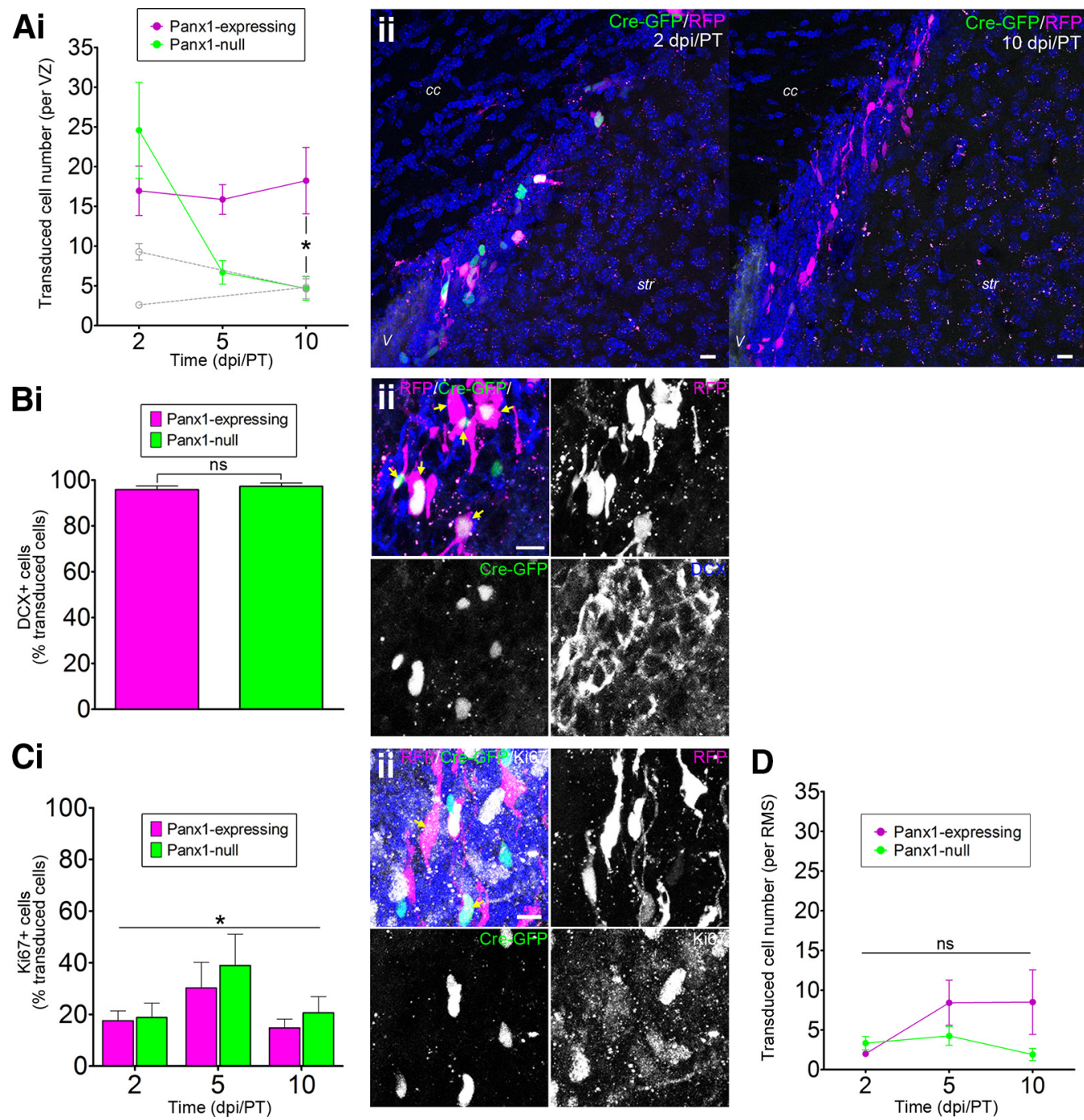

Figure 3. Panx1 is essential for maintaining elevated VZ NPC numbers after stroke. Ai, Panx1-null and Panx1-expressing NPC numbers per VZ at 2, 5 , and $10 \mathrm{dpi} / \mathrm{PT}$. Naive/sham data from Figure $2 A$ are overlaid in light gray. The number of Panx1-null NPCs significantly decreased over time ( $2 \mathrm{dpi} / \mathrm{PT}, n=7 ; 5$ and $10 \mathrm{dpi} / \mathrm{PT}, n=6$, expression: $F_{(1,32)}=2.854, p=$ 0.1008; time: $F_{(2,32)}=4.644, p=0.0169$; interaction: $F_{(2,32)}=4.902, p=0.0139$ by 2 -factor ANOVA; Bonferroni post hoc ${ }^{*} p<0.05$ for expression at 10 dpi/PT). Aii, Maximumintensity projections of representative confocal Z-stacks of the VZ show transduced NPCs at 2 (left) and $10 \mathrm{dpi} / \mathrm{PT}$ (right). Scale bars, $10 \mu \mathrm{m}$. V, Ventricle; cc, corpus callosum; str, striatum. Hoechst 33342 was used as a nuclear counterstain. Bi, The vast majority of transduced VZ NPCs were immunoreactive for DCX ( $2 \mathrm{dpi} / \mathrm{PT}, n=6, p=0.5202$ by unpaired $t$ test). Bii, Maximum-intensity projection of a representative confocal Z-stack from the VZ demonstrating DCX-immunoreactivity of transduced VZ NPCs. Scale bar, $10 \mu \mathrm{m}$. Arrows indicate DCX ${ }^{+}$ transduced NPCs. Ci, The percentage of transduced VZ NPCs immunoreactive for Ki67 was not affected by Panx1 expression status, but was affected by time after stroke (2 dpi/PT, $n=$ 7,5 and $10 \mathrm{dpi} / \mathrm{PT}, n=5$; expression: $F_{(1,28)}=0.8049, p=0.3760$; time: $F_{(2,28)}=3.461,{ }^{*} p=0.0454$; interaction: $F_{(2,28)}=0.1508, p=0.8607$ by 2-factor ANOVA). Cii, Maximum-intensity projection of a representative confocal Z-stack demonstrating Ki67 immunoreactivity of transduced VZ NPCs. Scale bar, $10 \mu \mathrm{m}$. Arrows indicate Ki67 ${ }^{+}$-transduced NPCS. Hoechst 33342 was used as a nuclear counterstain. D, Quantification of Panx1-null and Panx1-expressing NPC numbers in the RMS at 2,5, and $10 \mathrm{dpi} / \mathrm{PT}$. The number of transduced NPCs was not affected by Panx 1 expression status and did not significantly change over time ( 2 and $5 \mathrm{dpi} / \mathrm{PT}, n=6 ; 10 \mathrm{dpi} / \mathrm{PT}, n=5 ;$ expression: $F_{(1,28)}=3.593, p=0.0684 ;$ time: $F_{(2,28)}=1.812, p=0.1820$; interaction: $F_{(2,28)}=1.998, p=0.1545$ by 2 -factor ANOVA).

review, see Ohab and Carmichael, 2008) because Panx1 has been implicated in neuronal death (for review, see Weilinger et al., 2013) and inflammatory signaling (for review, see Makarenkova and Shestopalov, 2014) that persists in the peri-infarct cortex for days after the acute ischemic event (for review, see Brouns and De Deyn, 2009). There was a significantly greater abundance of Panx1-null NPCs in the peri-infarct cortex at $5 \mathrm{dpi} / \mathrm{PT}$ that persisted at $10 \mathrm{dpi} / \mathrm{PT}$ (Fig. 4A,B). This increase in Panx1-null NPCs in the peri-infarct cortex was not likely due to altered migration given that, at $2 \mathrm{dpi} / \mathrm{PT}$, there was not a surge of Panx1-null NPCs into the peri-infarct cortex nor the RMS. Analysis of the expression of activated caspase 3 within the transduced peri-infarct NPCs suggested that a relatively low percentage of these NPCs $(<10 \%)$ were apoptotic (Fig. $4 C)$. Our interpretation of these results is that Panx1-null NPCs persist longer in the peri-infarct cortex.

\section{Discussion}

Here, we examined the impact of the deletion of Panxl in VZ NPCs in the context of the healthy (naive/sham) and stroke-injured brain. This study builds on our recent discovery of Panxl expression in Nestin-positive/glial fibrillary acidic protein (GFAP)-positive, Nestin-positive/GFAP-negative (Wicki-Stordeur et al., 2012), and DCX-positive (Wicki-Stordeur and Swayne, 2013) VZ NPCs. Our previous results demonstrated that blocking Panxl channels in primary VZ NPC cultures reduced the number of VZ NPCs (WickiStordeur et al., 2012), suggesting that Panxl is involved in the regulation of their proliferation and/or maintenance in vitro. 


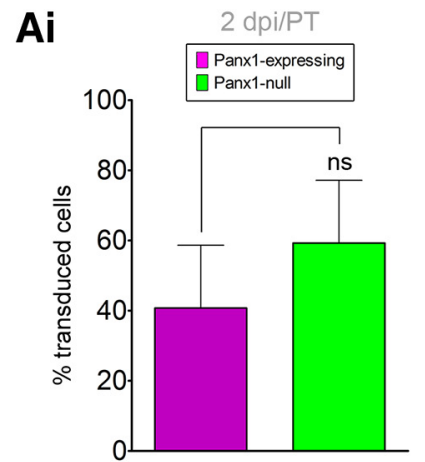

B

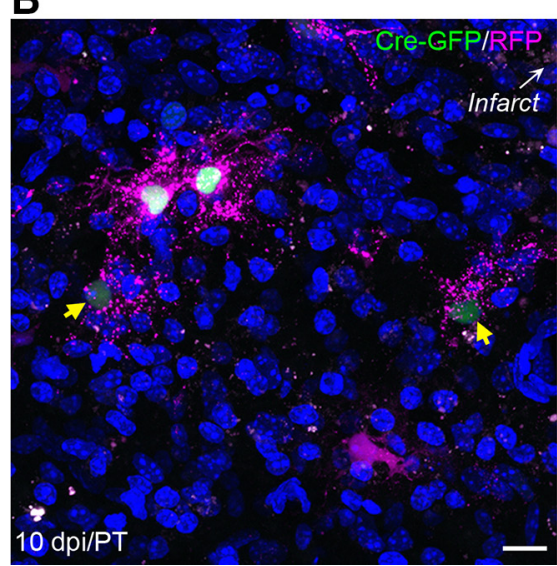

ii

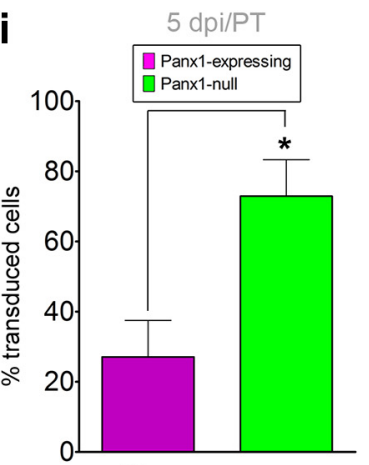

C
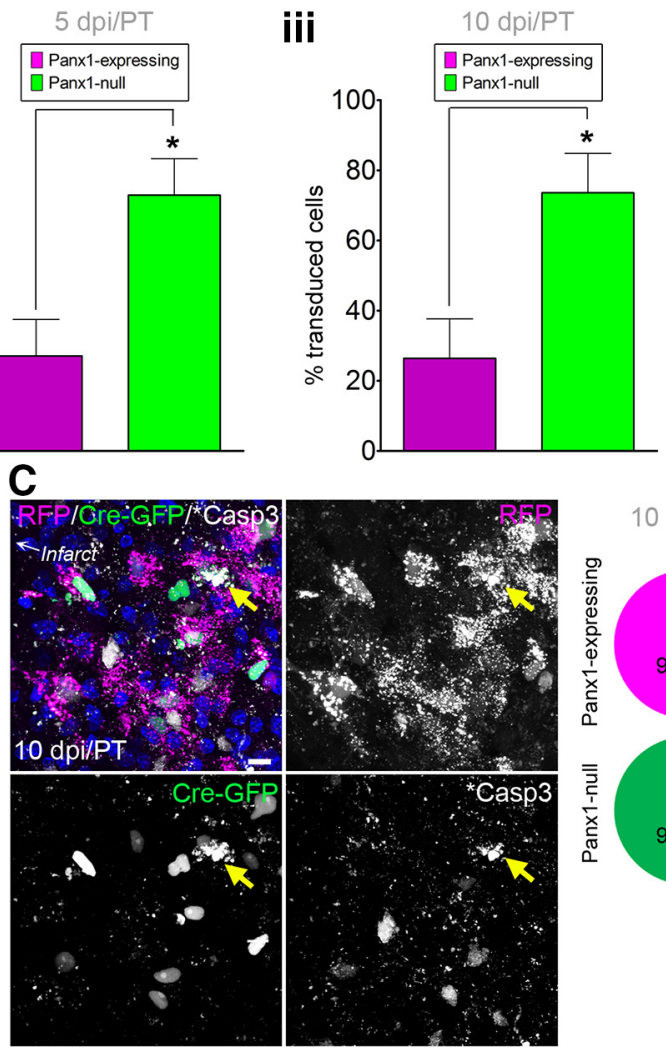

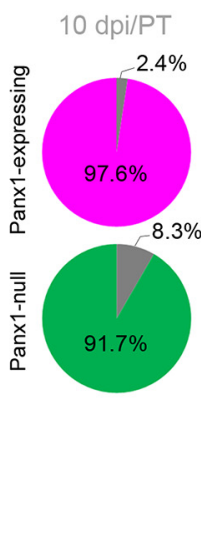

Figure 4. Panx1-null NPCs persist in the peri-infarct cortex. A, Percentages of Panx1-null and Panx1-expressing NPCs in the peri-infarct cortex. Ai, Panx1-null and Panx1-expressing NPCs were equally abundant at $2 \mathrm{dpi} / \mathrm{PT}(n=6, p=0.4812$ by unpaired $t$ test). Note that 1 of the 7 brains did not have a single transduced NPC in the peri-infarct at $2 \mathrm{dpi} / \mathrm{PT}$; data are represented as percentage of total transduced NPCs due to a large variability in NPC number reaching the peri-infarct cortex between mice. Aii, Panx1-null and Panx1-expressing NPC percentages at 5 dpi/PT ( $n=4, p=$ 0.0208 by unpaired $t$ test). Note that 2 of the 6 brains did not have a single transduced NPC in the peri-infarct at 5 dpi/PT. Aiii, Panx1-null NPCs were more abundant than Panx1-expressing NPCs at $10 \mathrm{dpi} / \mathrm{PT}$ ( $n=6, p=0.0180$ by unpaired $t$ test). $\boldsymbol{B}$, Maximum-intensity projection of a representative confocal Z-stack from the peri-infarct tissue $10 \mathrm{dpi} / \mathrm{PT}$. Arrows indicate faint GFP ${ }^{+}{ }_{\text {nuclei. } \boldsymbol{C} \text {, }}$ Maximum-intensity projection of a representative confocal Z-stack from the peri-infarct tissue $10 \mathrm{dpi} / \mathrm{PT}$ with yellow arrows indicating activated caspase $3\left({ }^{*}\right.$ Casp3) ${ }^{+}$cells. Pie charts indicate the percentage of total RFP ${ }^{+}$(Panx1-expressing; top) or GFP ${ }^{+}$(Panx1-null; bottom) cells that were ${ }^{*}$ Casp $3^{+}$in the peri-infact across all animals. Hoechst 33342 was used as a nuclear counterstain. Scale bars, $10 \mu \mathrm{m}$.

To test the hypothesis that Panxl regulates NPCs in vivo in the context of the healthy adult brain, we used a retrovirus-mediated approach to selectively delete Panx1 in late-stage VZ NPCs in floxed Panx1 mice. Initially, there were $70 \%$ fewer Panx1-null NPCs than Panx1-expressing NPCs in the VZ (2 dpi; Fig. 2). Our analyses suggested that Panx1 is required for maintenance of VZ NPCs through a mechanism other than proliferation, differentiation, migration, or apoptosis.

How does Panx 1 promote NPC maintenance in the VZ niche? In the VZ of the healthy brain, a large percentage of NPCs are normally lost (Morshead and van der Kooy, 1992). Recent evidence shows that these NPCs are cleared by neighboring NPCs (DCX-positive neuroblasts), which are the primary phagocytic cells in the VZ (Lu et al., 2011). Further work has shown that this phagocytic process in NPCs is likely regulated by a noncanonical $\mathrm{P}_{2} \mathrm{X}_{7}$-dependent mechanism that is inhibited in the presence of ATP (Lovelace et al., 2015). In this mechanism established in vitro and in vivo by $\mathrm{Gu}$ and colleagues (Gu et al., 2009, 2010, 2011, 2012), $P 2 X_{7}$ is required for phagocytosis, but when activated in the presence of ATP, it dissociates from its binding partner nonmuscle myosin, thereby abolishing further $\mathrm{P}_{2} \mathrm{X}_{7}$-mediated phagocytosis (for review, see Wiley and Gu, 2012). This suggests that ATP could act as a survival ("don't-eat-me") signal in addition to its previously defined roles in purinergic-receptormediated regulation of NPC maintenance and differentiation (for review, see Cavaliere et al., 2015). In other words, deletion of
Panx1 (a well known ATP-release channel) rendered cells vulnerable to "premature" clearance by resident phagocytic NPCs in a process termed "phagoptosis" (for review, see Brown and Neher, 2012 and Brown et al., 2015), resulting in the low abundance of Panx1-null NPCs in the healthy VZ.

Panx1-null cells were more abundant in the peri-infarct cortex $(5$ and $10 \mathrm{dpi} / \mathrm{PT})$ and a low percentage $(<10 \%)$ of transduced NPCs demonstrated signs of apoptosis, suggesting that the loss of Panx1 improved NPC maintenance. Overall, we did not observe a large number of activated caspase 3-positive cells in general, suggesting that apoptosis is not the primary mediator of NPC death in the peri-infarct cortex at this delayed period (days) after stroke. In fact, recent data suggest that the death of vulnerable neurons in the peri-infarct cortex occurs due to phagoptosis of viable cells exposed to sublethal stimuli (Neher et al., 2013). These cells present "find-me/eat-me" signals that attract phagocytic microglia (Neher et al., 2011; Neniskyte et al., 2011; GeigerMaor et al., 2012; Neher et al., 2013). Find-me/eat-me signals for microglia include things such as phosphatidylserine externalization and release of ATP (for review, see Patel et al., 2013). So how does Panx1 deletion factor in? In the peri-infarct cortex, ATP activates $\mathrm{P}_{2} \mathrm{Y}_{12}$ receptors expressed on microglia to elicit chemotaxis and phagocytosis of the target cell (Honda et al., 2001; Irino et al., 2008; Ohsawa et al., 2010). Therefore, the presence of Panx1 in NPCs in the peri-infarct would render NPCs vulnerable to phagoptosis. This is in stark contrast to ATP-mediated inhibi- 
tion of the noncanonical $\mathrm{P} 2 \mathrm{X}_{7}$-dependent NPC-mediated NPC phagocytosis in the VZ. Supporting this idea, ATP release through Panx1 was recently reported to be the "find-me" signal for phagocytic macrophages (Chekeni et al., 2010).

Promoting the survival of NPCs in the peri-infarct cortex has been associated with improved stroke outcomes (for review, see Xiong et al., 2010). Our results suggest that targeting Panx1 in the peri-infarct cortex, in combination with other therapies, could improve cell survival around the injury site. Tracking Panx1-null peri-infarct NPCs over a longer time course will be required to fully address the effects of Panxl on peri-infarct NPC survival and their impact on stroke outcomes.

As expected, based on previous studies that demonstrated stroke increases numbers of VZ NPCs (for review, see Ohab and Carmichael, 2008), we observed an increase in the number of transduced/labeled VZ NPCs after stroke. Similar to a previous report demonstrating bilateral NPC responses to focal stroke (Jin et al., 2001), we observed bilateral increases in labeled VZ NPC numbers. Elevated NPC numbers can persist for weeks and even months after stroke (for review, see Ohab and Carmichael, 2008). Our data suggested that Panx1 was required for the persistent elevation of NPC numbers since Panx1-null NPCs were significantly less abundant at $10 \mathrm{dpi} / \mathrm{PT}$ (Fig. 3). Compared with the healthy (naive/sham) brain scenario (where Panxl deletion led to an immediate loss in cells at $2 \mathrm{dpi}$ ), it appeared that the effect of Panxl deletion on VZ NPCs was masked and/or delayed by stroke. One aspect that our study did not address was whether Panx1 plays a role in stroke-mediated activation of NPCs. We induced stroke at the same time as retrovirus injection, so the initial stroke stimulus preceded actual decreases in Panx1 expression. Additional studies with Panx1 deletion before stroke will be needed to determine whether Panx1 plays a role in the initial activation of NPCs after stroke.

In summary, our observations reveal a new role for Panx 1 in NPC maintenance in the VZ and support the growing body of literature (for review, see Dahl and Keane, 2012), suggesting that targeting peri-infarct Panx1 (in combination with other interventions) could improve outcomes after stroke.

\section{References}

Bao L, Locovei S, Dahl G (2004) Pannexin membrane channels are mechanosensitive conduits for ATP. FEBS Lett 572:65-68. CrossRef Medline

Bargiotas P, Krenz A, Hormuzdi SG, Ridder DA, Herb A, Barakat W, Penuela S, von Engelhardt J, Monyer H, Schwaninger M (2011) Pannexins in ischemia-induced neurodegeneration. Proc Natl Acad Sci U S A 108: 20772-20777. CrossRef Medline

Bargiotas P, Krenz A, Monyer H, Schwaninger M (2012) Functional outcome of pannexin-deficient mice after cerebral ischemia. Channels (Austin) 6: 453-456. CrossRef Medline

Brouns R, De Deyn PP (2009) The complexity of neurobiological processes in acute ischemic stroke. Clin Neurol Neurosurg 111:483-495. CrossRef Medline

Brown GC, Neher JJ (2012) Eaten alive! Cell death by primary phagocytosis: 'phagoptosis'. Trends Biochem Sci 37:325-332. CrossRef Medline

Brown GC, Vilalta A, Fricker M (2015) Phagoptosis-cell death by phagocytosis-plays central roles in physiology, host defense and pathology. Curr Mol Med 15:842-851. CrossRef Medline

Bruzzone R, Hormuzdi SG, Barbe MT, Herb A, Monyer H (2003) Pannexins, a family of gap junction proteins expressed in brain. Proc Natl Acad Sci U S A 100:13644-13649. CrossRef Medline

Cavaliere F, Donno C, D’Ambrosi N (2015) Purinergic signaling: a common pathway for neural and mesenchymal stem cell maintenance and differentiation. Front Cell Neurosci 9:211. Medline

Chekeni FB, Elliott MR, Sandilos JK, Walk SF, Kinchen JM, Lazarowski ER, Armstrong AJ, Penuela S, Laird DW, Salvesen GS, Isakson BE, Bayliss DA, Ravichandran KS (2010) Pannexin 1 channels mediate 'find-me' signal release and membrane permeability during apoptosis. Nature 467: 863-867. CrossRef Medline

Dahl G, Keane RW (2012) Pannexin: from discovery to bedside in 11+/-4 years? Brain Res 1487:150-159. CrossRef Medline

Dvoriantchikova G, Ivanov D, Barakat D, Grinberg A, Wen R, Slepak VZ, Shestopalov VI (2012) Genetic ablation of Pannexin1 protects retinal neurons from ischemic injury. PLoS One 7:e31991. CrossRef Medline

Geiger-Maor A, Levi I, Even-Ram S, Smith Y, Bowdish DM, Nussbaum G, Rachmilewitz J (2012) Cells exposed to sublethal oxidative stress selectively attract monocytes/macrophages via scavenger receptors and MyD88-mediated signaling. J Immunol 188:1234-1244. CrossRef Medline

Gu BJ, Rathsam C, Stokes L, McGeachie AB, Wiley JS (2009) Extracellular ATP dissociates nonmuscle myosin from $\mathrm{P} 2 \mathrm{X}(7)$ complex: this dissociation regulates P2X(7) pore formation. Am J Physiol Cell Physiol 297: C430-C439. CrossRef Medline

Gu BJ, Saunders BM, Jursik C, Wiley JS (2010) The P2X7-nonmuscle myosin membrane complex regulates phagocytosis of nonopsonized particles and bacteria by a pathway attenuated by extracellular ATP. Blood 115: 1621-1631. CrossRef Medline

Gu BJ, Saunders BM, Petrou S, Wiley JS (2011) P2X(7) is a scavenger receptor for apoptotic cells in the absence of its ligand, extracellular ATP. J Immunol 187:2365-2375. CrossRef Medline

Gu BJ, Duce JA, Valova VA, Wong B, Bush AI, Petrou S, Wiley JS (2012) $\mathrm{P} 2 \mathrm{X} 7$ receptor-mediated scavenger activity of mononuclear phagocytes toward non-opsonized particles and apoptotic cells is inhibited by serum glycoproteins but remains active in cerebrospinal fluid. J Biol Chem 287; 17318-17330. CrossRef Medline

Honda S, Sasaki Y, Ohsawa K, Imai Y, Nakamura Y, Inoue K, Kohsaka S (2001) Extracellular ATP or ADP induce chemotaxis of cultured microglia through Gi/o-coupled P2Y receptors. J Neurosci 21:1975-1982. Medline

Irino Y, Nakamura Y, Inoue K, Kohsaka S, Ohsawa K (2008) Akt activation is involved in P2Y12 receptor-mediated chemotaxis of microglia. J Neurosci Res 86:1511-1519. CrossRef Medline

Jin K, Minami M, Lan JQ, Mao XO, Batteur S, Simon RP, Greenberg DA (2001) Neurogenesis in dentate subgranular zone and rostral subventricular zone after focal cerebral ischemia in the rat. Proc Natl Acad Sci U S A 98:4710-4715. CrossRef Medline

Locovei S, Wang J, Dahl G (2006) Activation of pannexin 1 channels by ATP through P2Y receptors and by cytoplasmic calcium. FEBS Lett 580: 239-244. CrossRef Medline

Lovelace MD, Gu BJ, Eamegdool SS, Weible MW 2nd, Wiley JS, Allen DG, Chan-Ling T (2015) P2X7 receptors mediate innate phagocytosis by human neural precursor cells and neuroblasts. Stem Cells 33:526-541. CrossRef Medline

Lu Z, Elliott MR, Chen Y, Walsh JT, Klibanov AL, Ravichandran KS, Kipnis J (2011) Phagocytic activity of neuronal progenitors regulates adult neurogenesis. Nat Cell Biol 13:1076-1083. CrossRef Medline

Ma W, Hui H, Pelegrin P, Surprenant A (2009) Pharmacological characterization of pannexin-1 currents expressed in mammalian cells. J Pharmacol Exp Ther 328:409-418. CrossRef Medline

Mak GK, Weiss S (2010) Paternal recognition of adult offspring mediated by newly generated CNS neurons. Nat Neurosci 13:753-758. CrossRef Medline

Makarenkova HP, Shestopalov VI (2014) The role of pannexin hemichannels in inflammation and regeneration. Front Physiol 5:63. Medline

Ming GL, Song H (2011) Adult neurogenesis in the mammalian brain: significant answers and significant questions. Neuron 70:687-702. CrossRef Medline

Morshead CM, van der Kooy D (1992) Postmitotic death is the fate of constitutively proliferating cells in the subependymal layer of the adult mouse brain. J Neurosci 12:249-256. Medline

Neher JJ, Neniskyte U, Zhao JW, Bal-Price A, Tolkovsky AM, Brown GC (2011) Inhibition of microglial phagocytosis is sufficient to prevent inflammatory neuronal death. J Immunol 186:4973-4983. CrossRef Medline

Neher JJ, Emmrich JV, Fricker M, Mander PK, Théry C, Brown GC (2013) Phagocytosis executes delayed neuronal death after focal brain ischemia. Proc Natl Acad Sci U S A 110:E4098-E4107. CrossRef Medline

Neniskyte U, Neher JJ, Brown GC (2011) Neuronal death induced by nanomolar amyloid beta is mediated by primary phagocytosis of neurons by microglia. J Biol Chem 286:39904-39913. CrossRef Medline

Ohab JJ, Carmichael ST (2008) Poststroke neurogenesis: emerging princi- 
ples of migration and localization of immature neurons. Neuroscientist 14:369-380. CrossRef Medline

Ohsawa K, Irino Y, Sanagi T, Nakamura Y, Suzuki E, Inoue K, Kohsaka S (2010) P2Y12 receptor-mediated integrin-betal activation regulates microglial process extension induced by ATP. Glia 58:790-801. Medline

Panchin Y, Kelmanson I, Matz M, Lukyanov K, Usman N, Lukyanov S (2000) A ubiquitous family of putative gap junction molecules. Curr Biol 10:R473-R474. CrossRef Medline

Patel AR, Ritzel R, McCullough LD, Liu F (2013) Microglia and ischemic stroke: a double-edged sword. Int J Physiol Pathophysiol Pharmacol 5:73-90. Medline

Ray A, Zoidl G, Weickert S, Wahle P, Dermietzel R (2005) Site-specific and developmental expression of pannexin1 in the mouse nervous system. Eur J Neurosci 21:3277-3290. CrossRef Medline

Sakamoto M, Ieki N, Miyoshi G, Mochimaru D, Miyachi H, Imura T, Yamaguchi M, Fishell G, Mori K, Kageyama R, Imayoshi I (2014) Continuous postnatal neurogenesis contributes to formation of the olfactory bulb neural circuits and flexible olfactory associative learning. J Neurosci 34: 5788-5799. CrossRef Medline

Santiago MF, Veliskova J, Patel NK, Lutz SE, Caille D, Charollais A, Meda P, Scemes E (2011) Targeting pannexin1 improves seizure outcome. PLoS One 6:e25178. CrossRef Medline

Scholzen T, Gerdes J (2000) The Ki-67 protein: from the known and the unknown. J Cell Physiol 182:311-322. CrossRef Medline

Silverman WR, de Rivero Vaccari JP, Locovei S, Qiu F, Carlsson SK, Scemes E, Keane RW, Dahl G (2009) The pannexin 1 channel activates the inflammasome in neurons and astrocytes. J Biol Chem 284:18143-18151. CrossRef Medline

Sosinsky GE, Boassa D, Dermietzel R, Duffy HS, Laird DW, MacVicar B, Naus CC, Penuela S, Scemes E, Spray DC, Thompson RJ, Zhao HB, Dahl G (2011) Pannexin channels are not gap junction hemichannels. Channels (Austin) 5:193-197. CrossRef Medline

Swayne LA, Sorbara CD, Bennett SA (2010) Pannexin 2 is expressed by postnatal hippocampal neural progenitors and modulates neuronal commitment. J Biol Chem 285:24977-24986. CrossRef Medline

Tashiro A, Zhao C, Gage FH (2006a) Retrovirus-mediated single-cell gene knockout technique in adult newborn neurons in vivo. Nat Protoc 1:3049-3055. Medline

Tashiro A, Sandler VM, Toni N, Zhao C, Gage FH (2006b) NMDA- receptor-mediated, cell-specific integration of new neurons in adult dentate gyrus. Nature 442:929-933. CrossRef Medline

Thompson RJ, Zhou N, MacVicar BA (2006) Ischemia opens neuronal gap junction hemichannels. Science 312:924-927. CrossRef Medline

Thornberry NA, Lazebnik Y (1998) Caspases: enemies within. Science 281: 1312-1316. CrossRef Medline

Vogt A, Hormuzdi SG, Monyer H (2005) Pannexin1 and Pannexin2 expression in the developing and mature rat brain. Brain Res Mol Brain Res 141:113-120. CrossRef Medline

Watson BD, Dietrich WD, Busto R, Wachtel MS, Ginsberg MD (1985) Induction of reproducible brain infarction by photochemically initiated thrombosis. Ann Neurol 17:497-504. CrossRef Medline

Weilinger NL, Tang PL, Thompson RJ (2012) Anoxia-induced NMDA receptor activation opens pannexin channels via Src family kinases. J Neurosci 32:12579-12588. CrossRef Medline

Weilinger NL, Maslieieva V, Bialecki J, Sridharan SS, Tang PL, Thompson RJ (2013) Ionotropic receptors and ion channels in ischemic neuronal death and dysfunction. Acta Pharmacol Sin 34:39-48. CrossRef Medline

Wicki-Stordeur LE, Swayne LA (2013) Panxl regulates neural stem and progenitor cell behaviours associated with cytoskeletal dynamics and interacts with multiple cytoskeletal elements. Cell Commun Signal 11:62. CrossRef Medline

Wicki-Stordeur LE, Dzugalo AD, Swansburg RM, Suits JM, Swayne LA (2012) Pannexin 1 regulates postnatal neural stem and progenitor cell proliferation. Neural Dev 7:11. CrossRef Medline

Wicki-Stordeur LE, Boyce AK, Swayne LA (2013) Analysis of a pannexin 2-pannexin 1 chimeric protein supports divergent roles for pannexin C-termini in cellular localization. Cell Commun Adhes 20:73-79. CrossRef Medline

Wiley JS, Gu BJ (2012) A new role for the P2X7 receptor: a scavenger receptor for bacteria and apoptotic cells in the absence of serum and extracellular ATP. Purinergic Signal 8:579-586. CrossRef Medline

Xiong XX, Gu LJ, Shen J, Kang XH, Zheng YY, Yue SB, Zhu SM (2014) Probenecid protects against transient focal cerebral ischemic injury by inhibiting HMGB1 release and attenuating AQP4 1 Neurochem Res 39:216-224.

Xiong Y, Mahmood A, Chopp M (2010) Angiogenesis, neurogenesis and brain recovery of function following injury. Curr Opin Investig Drugs 11:298-308. Medline 